\title{
Programmable Aperture Camera Using LCoS
}

\author{
Hajime Nagahara ${ }^{1}$, Changyin Zhou ${ }^{2}$, Takuya Watanabe ${ }^{3}$, Hiroshi Ishiguro ${ }^{3}$, \\ and Shree K. Nayar ${ }^{2}$ \\ 1 Kyushu University \\ 2 Columbia University \\ 3 Osaka University
}

\begin{abstract}
Since 1960s, aperture patterns have been studied extensively and a variety of coded apertures have been proposed for various applications, including extended depth of field, defocus deblurring, depth from defocus, light field acquisition, etc. Researches have shown that optimal aperture patterns can be quite different due to different applications, imaging conditions, or scene contents. In addition, many coded aperture techniques require aperture patterns to be temporally changed during capturing. As a result, it is often necessary to have a programmable aperture camera whose aperture pattern can be dynamically changed as needed in order to capture more useful information.

In this paper, we propose a programmable aperture camera using a Liquid Crystal on Silicon (LCoS) device. This design affords a high brightness contrast and high resolution aperture with a relatively low light loss, and enables one change the pattern at a reasonably high frame rate. We build a prototype camera and evaluate its features and drawbacks comprehensively by experiments. We also demonstrate two coded aperture applications in light field acquisition and defocus deblurring.
\end{abstract}

\section{Introduction}

In the past decades, coded aperture techniques have been studied extensively in optics, computer vision and computer graphics, and a variety of coded aperture techniques have been proposed for various applications. The optimal aperture patterns can be quite different from one application to another. For defocus deblurring, coded apertures are optimized to be broad-band in the Fourier domain [1] [2]. For depth from defocus, coded apertures are optimized to have more zerocrossing frequencies [3] [4]. For multiplexing light field acquisition, an optimal set of aperture patterns are solved for the best signal-to-noise ratio (SNR) after de-multiplexing [5]. Aperture can also be coded in the temporal dimension for motion deblurring [6]. Coded aperture methods have also been used in many other applications, including lensless imaging [7] [8], natural matting [9], etc. Figure 1 shows a collection of some coded apertures that were proposed in the past.

There are many situations where the aperture pattern should be dynamically updated as needed. First, from the aspect of information capturing, ideally aperture pattern should be adaptive to scene contents. For example, the pattern 


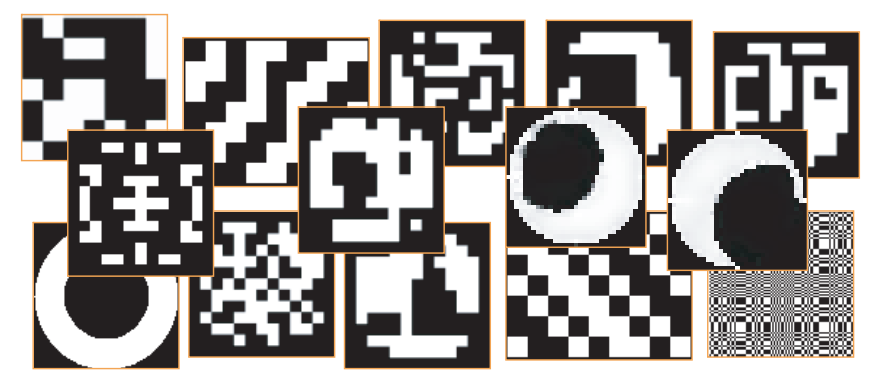

Fig. 1. A variety of coded aperture patterns proposed for various applications.

should be optimized for defocus deblurring if the scene has a large depth, and it should be optimized for motion deblurring if the scene has many objects in motion. Secondly, aperture pattern should be adaptive to the specific application purpose. For example, people have shown that a coded aperture optimized for defocus deblurring is often a bad choice for depth from defocus [4]; and multiplexing light field acquisition technique requires different aperture codings for different target angular resolutions. Thirdly, the pattern should be adaptive to the imaging condition. For example, the optimal aperture pattern for defocus deblurring is different at different image noise levels as shown in [2]. In addition, some coded aperture techniques need to capture multiple images with different aperture patterns (e.g., [6] [4] and [5]). In all these situations, people need a programmable aperture camera whose aperture pattern can be updated at a reasonable speed.

In literature, people has used transmissive liquid crystal displays (LCD) to control aperture patterns [8] [5]. However, the LCD implementation has severe drawbacks. The electronic elements on LCD pixels occlude lights and lead to a low light efficiency. These occluders also cause strong and complicated defocus and diffraction artifacts. These artifacts can be very strong and eliminate the benefits of aperture codings. Consider the popular applications of coded aperture (e.g., defocus deblurring, depth from defocus), we argue that a good programmable aperture is necessary to have the following features:

1. Easy mount. For different applications or scenes, people may use different lenses and sensors. Therefore, it is important to build a programmable aperture that can be easily mounted to different lenses and sensors.

2. High light efficiency. The loss of light leads to decreased SNR. As shown in [2] [10], a high light efficiency is the key to achieve high performance in defocus deblurring, depth from defocus, multiplexing light field acquisition, etc.

3. Reasonable frame rate. Some coded aperture techniques capture multiple images of a scene using different aperture patterns [4] [5]. For dynamic scenes, these techniques require multiple images to be captured within a reasonable short time in order to reduce motion blur, and at the same time, the aperture 
pattern must also be updated at the same frame rate and be synchronized with the sensor exposure.

4. High brightness contrast. Most optimized aperture patterns in literature have high brightness contrast - many of them are binary patterns. We may fail to display optimized patterns without a high brightness contrast.

To meet these requirements, we propose in this paper a programmable aperture camera by using a Liquid Crystal on Silicon (LCoS) device as shown in Figure 2. LCoS is a reflective liquid crystal device that has a high fill factor (92\%) and high reflectivity(60\%). Compared with transmissive LCD, an LCoS device usually suffers much less from light loss and diffraction. Figure 2 shows the structure of our proposed programmable aperture camera. The use of LCoS device in our prototype camera enables us to dynamically change aperture patterns as needed at a high resolution $(1280 \times 1024$ pixels $)$, a high frame rate $(5$ $\mathrm{kHz}$ maximum), and a high brightness contrast. By using the relay optics, we can mount any C-Mount or Nikkon F-Mount lens to our programmable aperture camera. Remarkably, our implementation used only off-the-shelf elements and people may reproduce or even improve the design for their own applications.

A detailed description and analysis to our proposed system will be given in Section 3. The features and limitations of the present prototype camera are evaluated via experiments in Section 4 . The proposed coded aperture camera can be a platform to implement many coded aperture techniques. As examples, in Section 5, we demonstrate the use of our prototype camera in two applications: multiplexing light field acquisition [5] and defocus deblurring [1] [2].

\section{Related work}

Coded aperture technique was first introduced in the field of high energy astronomy in 1960s as a novel way of improving SNR for lensless imaging of x-ray and $\gamma$-ray sources [11]. It is also in the 1960s that researchers in optics began developing unconventional apertures to capture high frequencies with less attenuation. In the following decades, many different aperture patterns were proposed (e.g., [12] [13] [14] [15] [7]).

Coded aperture research resurfaces in computer vision and graphics in recent years. People optimize coded aperture patterns to be broad-band in the Fourier domain in order that more information can be preserved during defocus for the later deblurring [1] [2]. Levin et al. [3] optimizes a single coded aperture to have more zero-crossing in the Fourier domain so that the depth information can be better encoded in a defocused image. Zhou et al. [4] show that by using the optimized coded aperture pair, they will be able to simultaneously recover a high quality focused image and a high quality depth map from a pair of defocused images. In the work [5], Liang et al. proposed to take a bunch of images using different coded aperture patterns in order to capture the light field.

Coded apertures have also been used for many other applications. Zomet and Nayar propose a lensless imaging technique by using an LCD aperture [16]. Raskar et al. uses a coded flutter shutter aperture for motion deblurring [6]. 


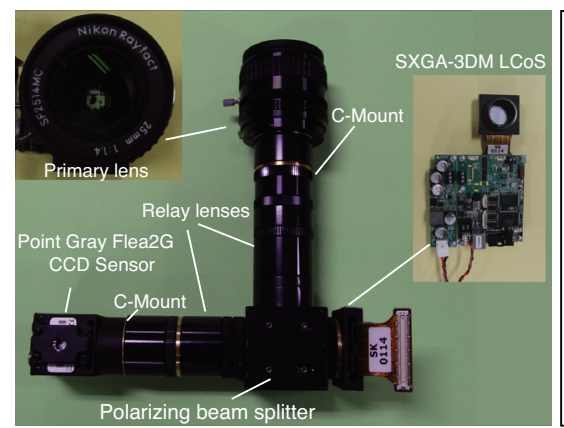

(a) Our prototype programmable aperture camera

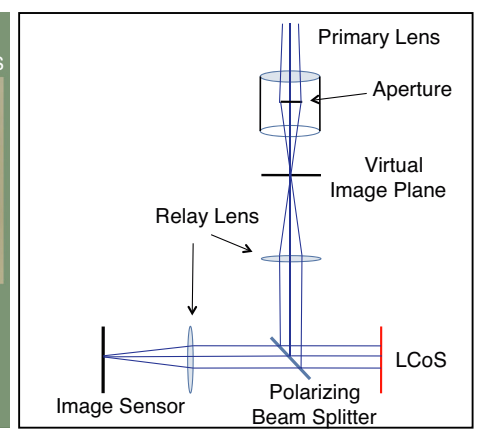

(b) The optical diagram of the prototype camera

Fig. 2. Programmable aperture camera using an LCoS device. (a) Our prototype LCoS programmable aperture camera. In the left-top corner is the Nikon F/1.4 25mm Cmount lens that is used in our experiments. On the right is an LCoS device. (b) The optical diagram of the proposed LCoS programmable aperture camera.

Coded aperture camera can be implemented in several ways. One popular coded aperture implementation is to disassemble the lens and insert a mask, which can be made of a printed film or even a cutted paper board [1] [3] [2]. The major disadvantages of this method are that one has to disassemble the lens, and that the pattern cannot be easily changed once the mask is inserted. Note that most commercial lenses cannot be easily disassembled without serious damages. People have also used some mechanical ways to modify apertures. Aggarwal and Ahuja propose to split the aperture by using a half mirror for high dynamic range imaging [17]. Green et al. build a complicated mechanical system and relay optics to split a circular aperture into three parts of different shapes [18].

To dynamically change aperture patterns during capturing, people has proposed to use transmissive liquid crystal display (LCD) devices as in the work [16] [5]. One problem with the LCD implementation is that the electronic elements sit in the LCD pixels not only block a significant portion of incoming light but also cause significant diffractions. Some custom LCDs are designed to have a higher light efficiency. However, these LCDs usually either have much low resolution (e.g., $5 \times 5$ pixels in [5]) or are prohibitively expensive. In this paper, we propose to use a reflective liquid crystal on silicon (LCoS) device [19], which has much higher light efficiency and suffers less from diffraction. LCoS has been used before in computer vision for high dynamic range imaging [20]. Another similar device that could be used to modulate apertures is the digital micro-mirror device (DMD). Nayar and Branzoi use a DMD device to control the irradiance to each sensor pixel for various applications, including high dynamic range and feature detection [21]. However, each DMD pixel only has two states and therefore DMD devices can only be used to implement binary patterns. 


\section{Optical Design and Implementation}

We propose to implement a programmable aperture camera by using a liquid crystal on silicon (LCoS) device as an aperture. LCoS is a reflective micro-display technique typically used in projection televisions. An LCoS device can change the polarization direction of rays that are reflected by each pixel. Compared with the typical transmissive LCD technique, it usually produces higher brightness contrast and higher resolution. Furthermore, LCoS suffers much less from light loss and diffraction than LCD does. This is because the electronic components sitting on each pixel of LCD device block lights and cause significant diffraction, and on the contrary, an LCoS device has all the electronic components behind the reflective surface and therefore provides much higher fill factors.

One of our major design goals is to make the primary lens separable from the programmable aperture in order that people can directly attach any compatible lenses without disassembling the lens. To achieve this, we propose to integrate an LCoS device into relay optics.

As shown in Figure 2, our proposed system consists of a primary lens, two relay lenses, one polarizing beam splitter, an LCoS device, and an image sensor. Only off-the-shelf elements are used in our prototype camera implementation. We choose a Forth dimension display SXGA-3DM LCoS micro-display. Table 1 shows the specifications of this LCoS device. We use two aspherical doublet lenses of 50mm focal length (Edmund Optics, part \#49665) for the relay optics, a cube polarizing beam splitter (Edmund Optics, part \#49002), and a Point Grey Flea2 camera (1/3" CCD, 1280x960 pixels at $25 \mathrm{fps})$. The camera shutter is synchronized with the LCoS device by using an output trigger $\left(25 \mathrm{~Hz}^{4}\right)$ of the LCoS driver.

People have a plenty of freedom in choosing primary lenses for this system. The primary lens and the image sensor are attached to the optics via the standard $\mathrm{C}$-mount. Therefore, a variety of C-mount cameras and lenses can be directly used with this prototype system. SLR lenses (e.g., Nikkon F-mount lenses) can also be used via a proper lens adopter. In our experiments, we use a Nikon $25 \mathrm{~mm}$ $\mathrm{F} / 1.4$ C-mount lens.

We can see from Figure 2 (b) that an incoming light from a scene is first collected by the primary lens and focused at the virtual image plane. A cone of light from each pixel of the virtual image plane is then forwarded by the first relay lens to the polarizing beam splitter. The beam splitter separates the light into Spolarized and P-polarized (perpendicular to each other) lights by reflection and transmission, respectively. The reflected S-polarized light is further reflected by LCoS. The LCoS device can rotate the polarization direction at every pixel by arbitary degrees. For example, if the pixel on LCoS is set to 255 (8bit depth), the polarization of the light is rotated by 90 degree and becomes P-polarized, and then the light will pass through the splitter and reach to the sensor. If the

\footnotetext{
${ }^{4}$ Note that the $\mathrm{LCoS}$ can be modulated at $5 \mathrm{kHz}$ maximum. We use $25 \mathrm{~Hz}$ in order that it can be synchronized with the sensor.
} 


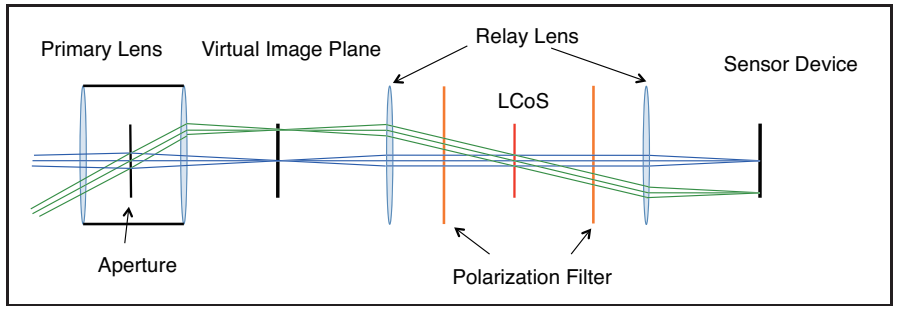

Fig. 3. An equivalent optical diagram to that in Figure 2 (b). The virtual image plane and the sensor plane are conjugated by the relay lens. The LCoS is the aperture stop of the system.

pixel on LCoS is set to 0 , the polarization will not be changed by LCoS and the reflected light will be blocked by the splitter.

Consider the LCoS device as a mirror, the diagram in Figure 2 (b) can be easily shown equivalent to that in Figure 3 . The proposed optics can be better understood from Figure 3. The sensor is located at the focal plane of the second relay lens, therefore the sensor plane is conjugate to the virtual image plane whose distance to the first relay lens is the focal length of the first relay lens. The LcoS device is relatively smaller than other stops in this optical system and works as the aperture stop.

\section{Optical Analysis and Experimental Evaluation}

Effective F-number Since the LCoS device works as the aperture stop in the proposed system, F-number $(f / \#)$ of the primary lens is no longer the effective $f / \#$ of the camera. The actual $f / \#$ of the system is decided by focal length of the relay lens $f_{r}$ and physical size of LCoS. For a circular aperture, $f / \#$ is usually defined as the ratio of focal length to the aperture diameter. For the rectangle nature of the LCoS, we use $2 \sqrt{u v / \pi}$ as the diameter, where $(\mathrm{u}, \mathrm{v})$ is the dimension of LCoS. Therefore have:

$$
f / \#=\frac{2}{f_{r}} \sqrt{\frac{u v}{\pi}} .
$$

According to Equation 1, the effective $f / \#$ of the prototype can be computed as $f / 2.84$, while the $f / \#$ of the primary lens is $f / 1.4$.

Field of View Figure 3 shows that the relay system copies the virtual image to sensor plane by a magnification ratio of $1: 1$. Therefore, the field of view (FOV) of the proposed camera is the same as if the sensor were placed at the virtual image plane. The FOV can be estimated by using the sensor size and the effective focal length of the primary lens:

$$
F O V \approx 2 \arctan \frac{d}{2 f_{p}}
$$


Table 1. Specification of LCoS device

\begin{tabular}{|l|r|}
\hline Resolution & $1280 \times 1024$ pixels \\
\hline Reflective depth & 8 bits \\
\hline Pixel fill factor & $>92 \%$ \\
\hline Reflectivity & $60 \%$ \\
\hline Contrast ratio & $400: 1$ \\
\hline Physical dimension & $17.43 \times 13.95 \mathrm{~mm}$ \\
\hline Switching pattern & $40 \mu \mathrm{s}$ \\
\hline
\end{tabular}

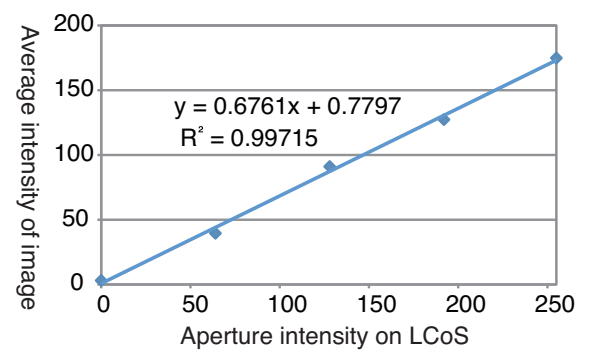

Fig. 4. The aperture transmittance is linear to the LCoS intensity.

where $d$ is a diagonal size of the sensor and $f_{p}$ is the effective focal length of the primary lens.

Our prototype camera uses a $25 \mathrm{~mm}$ lens and therefore the camera FOV can be computed as $13.69^{\circ}$ according to Equation 2. Of course, we can change the FOV by using a primary lens with a different focal length.

Light Efficiency Light efficiency is one of the most important index in a coded aperture camera. Ideally, the light efficiency of our prototype camera is calculated by:

$$
27.6 \%=50 \%\left(\text { polarization }^{5}\right) \times 92 \%(\text { fill factor }) \times 60 \%(\text { reflectivity }) .
$$

We notice that many other optical elements in the camera (e.g., a beam splitter, two relay lenses, and an LCoS device) may also attenuate the intensity of captured images. To measure the light efficiency accurately, we captured two images of a uniformly white plane. One image was captured using our prototype camera, and another image was captured without the LCoS aperture (the same sensor and the same lens with $f$ /\# set to 2.8). The ratio of the averaged brightness of these two captured images is computed as 41.54:202.0, which indicates the light efficiency of the system. The light efficiency of our system is about $21 \%$.

The theoretical light efficiency of a transmissive $\mathrm{LCD}{ }^{6}$ can also be calculated using a similar formula:

$$
7.4 \%=50 \%(\text { polarization }) \times 55 \%(\text { fill factor }) \times 27 \%(\text { transmittance }) .
$$

The light efficiency of our LCoS implementation is at least three times higher than that of the LCD implementation.

\footnotetext{
${ }^{5}$ A polarized beam splitter splits incoming lights based on their polarizations. Although the light interacts with the splitter twice, the light efficiency of beam splitter is still $50 \%$. This is because $100 \%$ light will pass through the splitter at the second interaction when its polarization is aligned to that of the splitter.

${ }^{6}$ Note that the fill factor or transmittance of the LCD can be slightly different due to different implementations (e.g., physical sizes and resolutions). We assume a typical LCD with a similar physical size and resolution to the LCoS used in our implementation.
} 


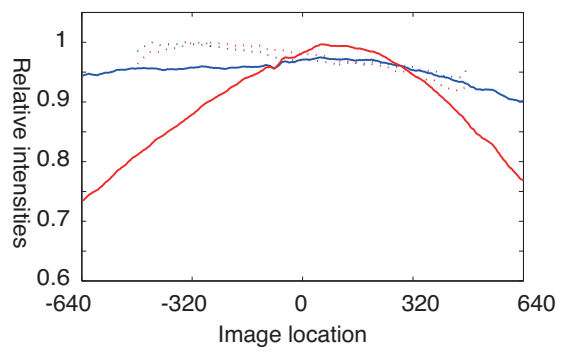

Fig. 5. Vignetting profiles. The red and blue solid lines indicate the horizontal vignetting curves of the prototype camera and a regular camera, respectively. The dashed lines indicate their vertical vignetting profiles.

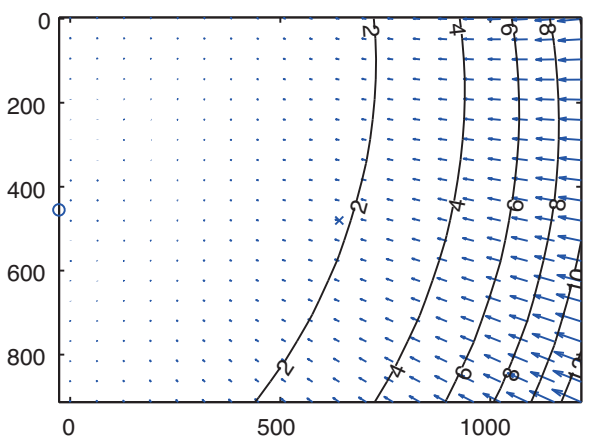

Fig. 6. Geometric distortion due to the use of doublet lenses.

Vignetting From the two images captured with and without the LCoS aperture, we can compute the vignetting curves of the prototype camera and a normal camera. The horizontal vignetting curves of our prototype camera and a normal camera are shown in Figure 5 in red and blue solid lines, respectively. The corresponding dashed lines show the vertical vignetting curves.

Transmission Fidelity Another important quality index of a coded aperture implementation is the transmission fidelity - the consistence between the actual transmittance of coded aperture and the input intensity of the LCoS device. To evaluate the transmission fidelity, we captured images of uniformly white plane using circular apertures of different intensities. Figure 4 shows the line of the average intensity of captured images with respect to the input intensities of the circular aperture (implemented using LCoS device). This plot confirms that the aperture intensity is linear to the actual light transmittance rate. Also, by a linear regression, we can calculate the maximum contrast ratio is 221:1. Although this contrast is not as high as in Table 1, it has been high enough for most coded aperture applications.

Distortion Another problem that has been caused by the use of doublets in the relay optics is image distortion. The geometric distortion is calibrated by using the Matlab camera calibration toolbox as shown in Figure 6. The circle indicates a center of distortion and the arrows represent displacements of the pixel introduced by the lens distortion. These calibrated camera parameters will be used to compensate the geometric distortions in the captured images.

PSF Evaluation Lens aberration and diffraction may distort the actual PSFs. To assess the PSF quality of the prototype camera, we display a coded aperture 


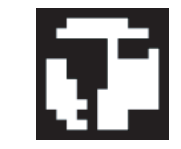

(a) The Input Aperture Pattern

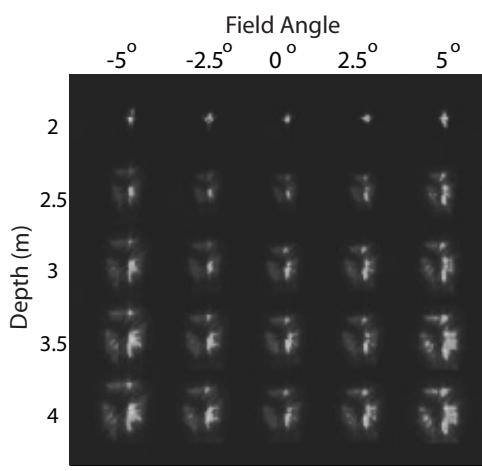

(b) The Calibrated PSFs

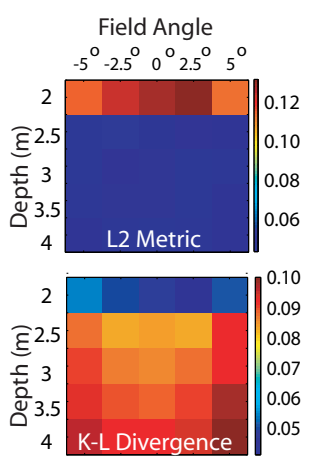

(c) PSF Dissimilarity Map

Fig. 7. Evaluating the PSFs of the prototype camera. (a) The coded aperture pattern used in the evaluation. This pattern is picked without specific intentions. (b) The calibrated PSFs at five depths ranging from $2 \mathrm{~m}$ to $4 \mathrm{~m}$, and five field angles ranging from $-5^{\circ}$ to $5^{\circ}$. We can see that the scale of PSFs varies with both depth and field angle (due to field curvature), while the shape of PSFs appears similar. (c) The shape dissimilarity between the input pattern and each PSF is computed according to two metrics: $L_{2}$ distance at the top, and K-L divergence at the bottom (as used in the work $[22])$.

Table 2. Specification of the prototype camera

\begin{tabular}{|c|c|}
\hline Image resolution & $1280 \times 960$ pixels \\
\hline Frame rate & $25 \mathrm{fps}$ \\
\hline Minimum F-number & 2.84 \\
\hline FOV(diagonal) & $13.76^{\circ}(25 \mathrm{~mm}$ Nikkon C-mount $)$ \\
\hline Actual aperture contrast & $221: 1$ \\
\hline Light transmittance & $20.56 \%$ \\
\hline
\end{tabular}

and then calibrate the camera PSFs at 5 depths and 5 different view angles. Without specific intentions, we use the aperture pattern as shown in Figure 7 (a) in this evaluation. Figure (b) shows how PSFs varies with depth and field angle. We can see that the scale of PSF is related to the field angle. This is because the use of doublets in the relay optics leads to a field curvature.

We can see that the shapes of most PSFs are still very similar. To measure the similarity between these PSFs and the input aperture pattern, we normalize the scale of each PSF and compute its $L_{2}$ distance to the input pattern. A distance map is shown in the top of Figure 7 (c). We can see that according to the $L_{2}$ distance, the PSF shape deviation decreases as the blur size increases. It is known that $L_{2}$ distance is not a good metric to measure the PSF similarities in defocus deblurring. To measure the dissimilarity between two PSFs, we use the Wiener reconstruction error when an image is blurred with one PSF and 
then deconvolved with another PSF. This reconstruction error turns out to be a variant of K-L divergence as shown in the work [22]. We plot this dissimilarity map in the bottom of Figure 7 (c). We can see that all the dissimilarity values are small and decrease as the blur size increases.

The specifications of the prototype programmable aperture camera are shown in Table 2 as a summary.

\section{Evaluation by Applications}

\subsection{Programmable Aperture for Light Field Acquisition}

We first use our prototype programmable aperture camera to re-implement the multiplexing light field acquisition method, which is first proposed by Liang et al. [5]. A 4D light field is often represented as $l(u, v, x, y)[23]$, where $(u, v)$ is the coordinates on the aperture plane and $(x, y)$ is the coordinates in the image plane.

For a light field acquisition technique using coded aperture, the spatial resolution in the $(x, y)$ space is simply determined by the sensor resolution and the angular resolution in the $(u, v)$ space is determined the resolution of coded apertures. Bando et al. [9] use a 2x2 color coded aperture to capture light fields and then use the information to do layer estimation and matting. Liang et al. [5] propose a multiplexing technique to capture light fields up to $7 \times 7$ angular resolution. For any $m \times n$ angular resolution light field acquisition, the multiplexing method requires $m \times n$ images captured using $m \times n$ different coded apertures.

With our prototype programmable aperture camera, it is easy to capture light fields with various angular resolutions. We use S-matrix for the multiplexing coding (see [24] for a deep discussion on the multiplexing coding). Figure 8 (top) shows four of the 31 aperture patterns ${ }^{7}$ that we generate from an S-Matrix. Since the aperture pattern of the prototype camera can be updated at a video frame rate ( $25 \mathrm{fps}$ ), it only takes 1.2 seconds to capture all of the images. If we could increase the camera frame rate further or lower the aperture resolution, the programmable aperture camera could be able to capture light fields of moving objects.

From the 31 captured images, we recover the light field of resolution $1280 \times$ $960 \times 31(7 \times 5(\mathrm{u}, \mathrm{v})$ resolution excluding the four corners $)$. Figure 9 shows the images for different viewpoints $(u, v)$ and their close-ups. From the close-ups, we can see the disparities of the text clearly. With the recovered light field, people will be able to do further post-processing including depth estimation and refocusing as shown in [5] and [9].

\subsection{Programmable Aperture for Defocus Deblurring}

Another important limit of most existing coded aperture implementations is that the actual shape of the produced PSF often deviates from the input pattern

\footnotetext{
7 This is because the code length of S-matrix must be $2^{n}-1$.
} 

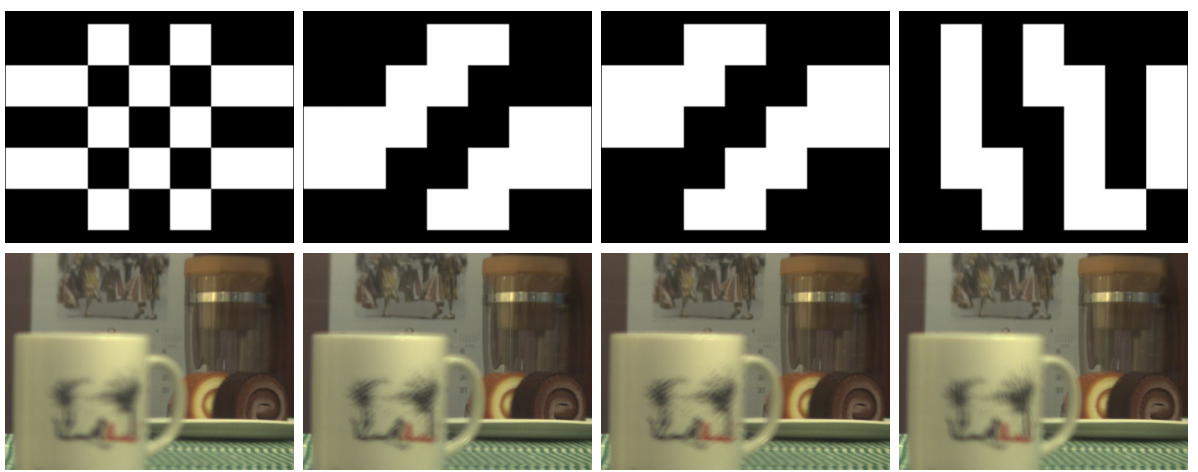

Fig. 8. Four multiplexing aperture codings and the corresponding captured images. Upper row shows four of the 31 aperture patterns that we generate from an S-Matrix. Bottom row shows the four corresponding captured images.

due to lens aberration and diffraction. Note that the effects of lens aberration and diffraction can be quite different in different lenses. For the complexity of the modern lenses, it is difficult to take these effects into account during pattern optimization. The effects of these imperfections on the optimality of the apertures are often overlooked in the literature.

With a programmable aperture camera, we will be able to evaluate the input aperture pattern by analyzing the captured images, and then improve the aperture patterns dynamically for a better performance. In this experiment, we apply this idea to the coded aperture technique for defocus deblurring.

Zhou and Nayar [2] propose a comprehensive criterion of aperture evaluation for defocus deblurring, which takes image noise level, the prior structure of natural images, and deblurring algorithm into account. They have also shown that the optimality of an aperture pattern can be different at different noise levels and scene settings. For a PSF $k$, its score at a noise level $\sigma$ is measured as:

$$
R(K \mid \sigma)=\Sigma \frac{\sigma^{2}}{|K|^{2}+\sigma^{2} /\left|F_{0}\right|^{2}},
$$

where $K$ is the Fourier transform of the PSF $k$, and $F_{0}$ is the Fourier transform of the ground truth focused image. This definition can be re-arranged as

$$
R(K \mid \sigma)=\Sigma \frac{\sigma^{2} \cdot\left|F_{0}\right|^{2}}{|K|^{2} \cdot\left|F_{0}\right|^{2}+\sigma^{2}} \approx \Sigma \frac{\sigma^{2} \cdot A}{|F|^{2}+\sigma^{2}} \propto \Sigma \frac{A}{|F|^{2}+\sigma^{2}},
$$

where $A$ is the average power spectrum of natural images as defined in the work [2], and $F$ is the Fourier transform of the captured image. Therefore, given a captured defocused image $F$, the equation 6 can be used to directly predict the quality of deblurring without calibrating the PSF and actually performing deblurring, while all the effects of aberrations and diffraction have been taken 


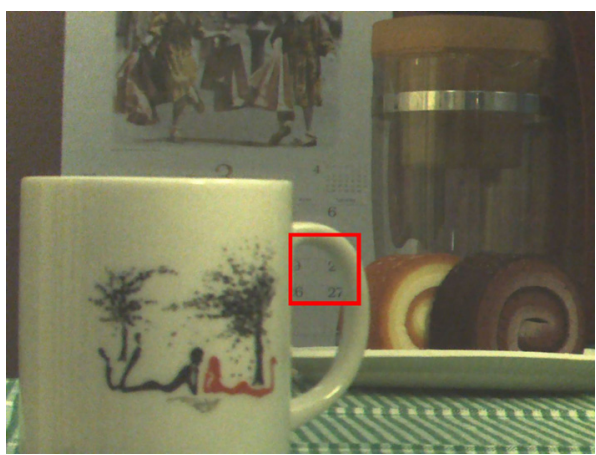

$(u, v)=(2,3)$

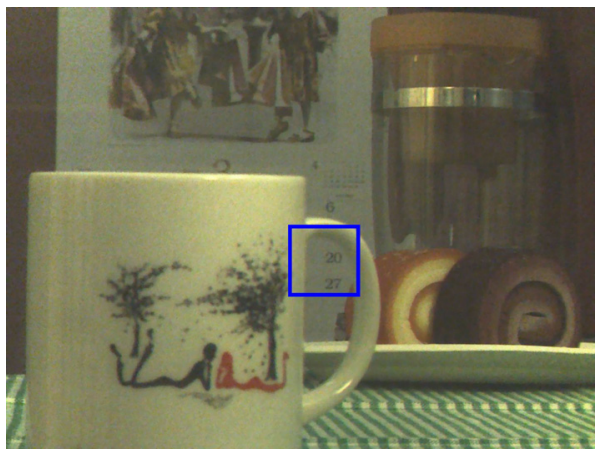

$(u, v)=(6,3)$

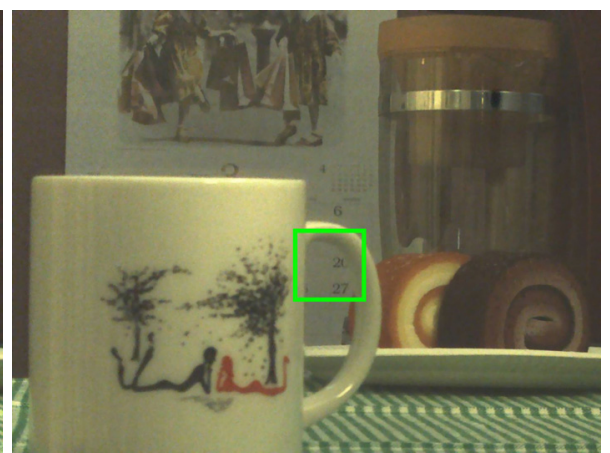

$(u, v)=(4,3)$

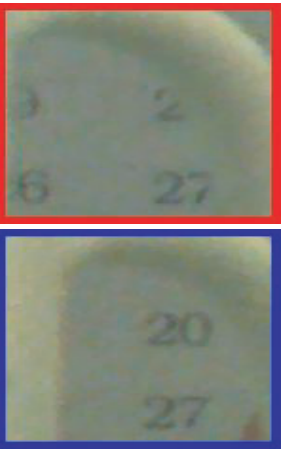

Close-up images

Fig. 9. The reconstructed 4D light field. Images from three different view points $(u, v)$ are generated from the reconstructed 4D light field, and their close-ups are shown in the right-bottom corner. From the close-up images, we can see the disparities of the text.

into account. Obviously, for the best deblurring quality, we should choose the aperture pattern which yields the lowest $R$ value.

In our experiment, we capture a set of defocused images of an IEEE resolution chart (shown in the first row of Figure 10) by using the aperture patterns shown in Figure 1. We compute the $R$ value from each captured image and find that the lowest $R$ value is achieved by using the pattern shown in Figure 10 (e). This indicates that this pattern is the best among all these candidate patterns in the present imaging condition and scene settings.

Note that this prediction is made directly from the observed defocused images without PSF calibration or deblurring. The computation only involves few basic arithmetic operations and one Fourier transform, and therefore can be done at real time. For comparison, the second row of Figure 10 shows the deblurring results of several different aperture patterns. These results confirm that the pattern in (e) is the best for defocus deblurring in this particular image condition. 


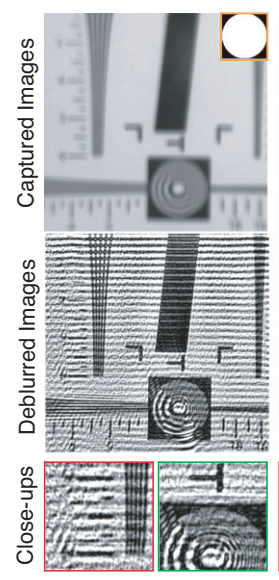

(a) Circular Pattern

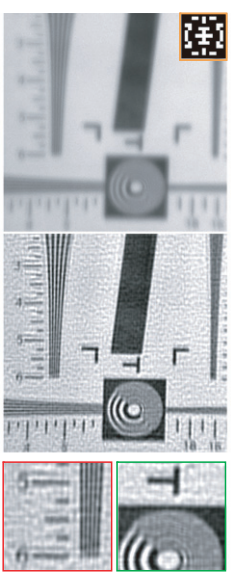

(b) Levin et al.

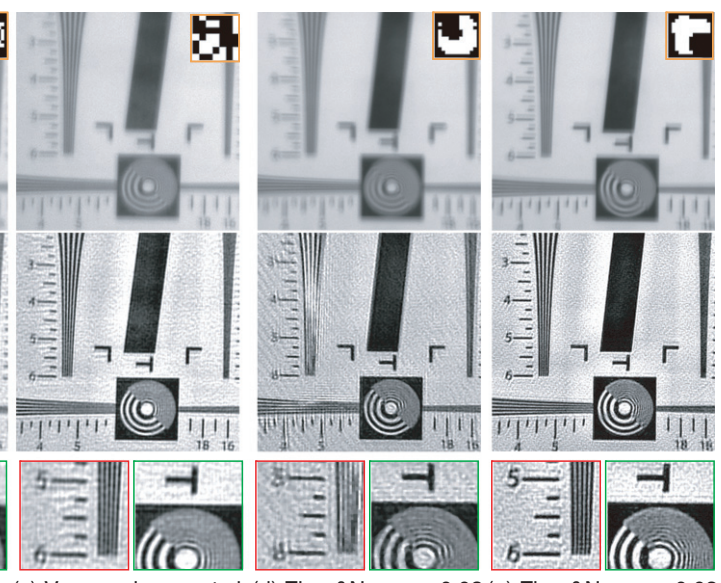

(c) Veeraraghavan et al. (d) Zhou\&Nayar $\sigma=0.02$ (e) Zhou\&Nayar $\sigma=0.03$

Fig. 10. Pattern selection for defocus deblurring by using the programmable aperture camera. We capture a set of defocused images of an IEEE resolution chart using the patterns shown in Figure 1, and evaluate their qualities using Equation 6. The pattern shown in Column (e) is found to be the best according to our proposed criterion. To verify this prediction, we calibrate the PSFs in all the captured images, do deblurring, and show deblurring results (the second and third rows). We can see that the deblurring result in Column (e) is the best, which is consistent with the prediction.

\section{Conclusion and Perspectives}

In this paper, we propose to build a programmable aperture camera using an LCoS device which enables us to implement aperture patterns of high brightness contrast, light efficient and resolution at a video frame rate. Another important feature of this design is that any C-Mount or F-Mount lenses can be easily attached to the proposed camera without being disassembled. These features make our design applicable to a variety of coded aperture techniques. We demonstrate the use of our proposed programmable aperture camera in two applications: multiplexing light field acquisition and pattern selection for defocus deblurring.

We are aware that our prototype camera has many imperfections. For example, using two doublets to relay the lights has led to severe lens aberration, vignetting, and field curvature; and the light efficiency of the prototype camera is lower than that in design. How to optimize the optical design to minimize these imperfections will be our future work.

\section{References}

1. Veeraraghavan, A., Raskar, R., Agrawal, A., Mohan, A., Tumblin, J.: Dappled photography: mask enhanced cameras for heterodyned light fields and coded aperture refocusing. ACM Trans. Graphics (2007) 
2. Zhou, C., Nayar, S.: What are good apertures for defocus deblurring? In: International Conference of Computational Photography, San Francisco, U.S. (2009)

3. Levin, A., Fergus, R., Durand, F., Freeman, W.: Image and depth from a conventional camera with a coded aperture. Proc. ACM SIGGRAPH 26 (2007) 70

4. Zhou, C., Lin, S., Nayar, S.: Coded Aperture Pairs for Depth from Defocus. In: Proc. International Conference on Computer Vision, Kyoto, Japan (2009)

5. Liang, C.K., Lin, T.H., Wong, B.Y., Liu, C., Chen, H.: Programmable aperture photography: Multiplexed light field acquisition. ACM Trans. Graphics 27 (2008)

6. Raskar, R., Agrawal, A., Tumblin, J.: Coded exposure photography: motion deblurring using fluttered shutter. ACM Trans. Graphics (2006) 795-804

7. Gottesman, S., Fenimore, E.: New family of binary arrays for coded aperture imaging. Applied Optics (1989) 4344-4352

8. Zomet, A., Nayar, S.: Lensless Imaging with a Controllable Aperture. In: Proceedings of the 2006 IEEE Computer Society Conference on Computer Vision and Pattern Recognition-Volume 1, IEEE Computer Society (2006) 346

9. Bando, Y., Chen, B., Nishita, T.: Extracting depth and matte using a color-filtered aperture. (2008)

10. Hasinoff, S., Kutulakos, K., Durand, F., Freeman, W.: Time-constrained photography. In: Proc. International Conference on Computer Vision. (2009) 1-8

11. Caroli, E., Stephen, J., Cocco, G., Natalucci, L., Spizzichino, A.: Coded aperture imaging in X-and gamma-ray astronomy. Space Science Reviews (1987) 349-403

12. Welford, W.: Use of annular apertures to increase focal depth. Journal of the Optical Society of America A (1960) 749-753

13. Mino, M., Okano, Y.: Improvement in the OTF of a defocused optical system through the use of shaded apertures. Applied Optics (1971) 2219-2225

14. Varamit, C., Indebetouw, G.: Imaging properties of defocused partitioned pupils. Journal of the Optical Society of America A (1985) 799-802

15. Ojeda-Castafieda, J., Andres, P., Diaz, A.: Annular apodizers for low sensitivity to defocus and to spherical aberration. Optics Letters (1986) 487-489

16. Zomet, A., Nayar, S.: Lensless imaging with a controllable aperture. Proc. Computer Vision and Pattern Recognition (2006) 339-346

17. Aggarwal, M., Ahuja, N.: Split Aperture Imaging for High Dynamic Range. International Journal of Computer Vision 58 (2004) 7-17

18. Green, P., Sun, W., Matusik, W., Durand, F.: Multi-aperture photography. Proc. ACM SIGGRAPH 26 (2007)

19. Wikipedia: Liquid crystal on silicon. (http://en.wikipedia.org/wiki/Liquid_ crystal_on_silicon)

20. Mannami, H., Sagawa, R., Mukaigawa, Y., Echigo, T., Yagi, Y.: High dynamic range camera using reflective liquid crystal. Proc. International Conference on Computer Vision (2007) 14-20

21. Nayar, S.K., Branzoi, V., Boult, T.: Programmable imaging: Towards a flexible camera. International Journal of Computer Vision 70 (2006) 7-22

22. Nagahara, H., Kuthirummal, S., Zhou, C., Nayar, S.: Flexible depth of field photography. In: Proc. European Conference on Computer Vision. Volume 3. (2008)

23. Levoy, M., Hanrahan, P.: Light field rendering. Proc. ACM SIGGRAPH (1996) $31-42$

24. Schechner, Y., Nayar, S., Belhumeur, P.: A theory of multiplexed illumination. Proc. International Conference on Computer Vision 2 (2003) 808-815 